unterscheiden und auch die kurzen Pycnoconidien geben ein gutes Unterscheidungsmerkmal.

Die Variationen der. Physcia ragusana sind analog derjenigen bei Physcia pulverulenta (Schreb.).

Physcia pulverulenta var. angustata Nyl. in Act. Soc. Linn. Bordeaux, vol. XXI (1856) pag. 308; Crombie, Monogr. Brit. Lich. vol. I (1894) pag. 307. - Lichen angustatus Hoffm., Enum. Lichen, Fasc. 1 (1784) Tab. XI, Fig. 2. - Parmelia pulverulenta $\gamma$. P. angustata Ach., Lichgr. Univ. (1810) pag. 474.

Bocche di Cattaro: bei Devesite, an Quercus, c. $750 \mathrm{~m}$ (Bau mgartner).

In den vorliegenden Stücken sind die Enden und hie und àa auch die Oberseite des Lagers in Form von Längsstreifen, dicht weiss bereift, was der Flechte ein ungemein zierliches Aussehen verleiht. Diese Bereifung erwähnt auch Acharius a. 0 . 0 , indem er sagt, dass die Varietät mit Ausnahme der Lappenbildung sich in nichts von der var. argyphaea unterscheidet, während $\mathrm{N}$ y lan d er hingegen die Varietät als unbereift beschreibt. 280. Physcia speciosa (Wulf.) Nyl.

Bocche di Cattaro: Devesite bei Castelnuovo, c. $750 \mathrm{~m}$, an Eichen (Baumgartner).

281. Anaptychia ciliaris (L.) Mass.

Halbinsel Lapad bei Ragusa, an Pinus halepensis (Ba u mgartner), in einer Form mit kurzbehaarter Lageroberseite; Bocche di Cattaro: Devesite bei Castelnuovo, c. $750 \mathrm{~m}$, an Eichen (Baumgartner).

\title{
Georg Treffer.
}

Das tirolische Pusterthal darf sich rühmen, die Heimat einer Reihe von Männern zu sein, deren Leistungen in der Beschaffung und Präparation von Herbarmaterial, dieser wichtigsten Grundlage floristischer Studien, die gerechte Bewunderung der Botaniker aller europäischen Länder erweckt haben.

Leider gehrrt diese einzig dastehende Schule nun schon sozusagen der Vergangenheit an: Ausserdorfer und Gander sind nicht mehr; Thomas Pichler sehen wir den Gebrechen des Alters verfallen und auch Rupert Huter, der zähe, unermüdliche, ist an einer Stufe des Lebensganges angelangt, welche physische Anstrengungen nicht mehr gestattet.

Dazu ist uns am 31. October 1902 das jüngste Mitglied dieses Kreises, Georg Treffer, durch den Tod entrissen worden.

Wohl wenigen der zahlreichen Besitzer seiner mustergiltig aufgelegten Exsiccaten mag etwas Näheres über den Lebensgang des merkwürdigen Mannes bekannt geworden sein und so schien es dem Unterzeichneten eine ebenso den Empfindungen der Pietät entsprechende als psychologisch interessante Aufgabe, zu ermitteln, in welcher Weise sich bei so anspruchslosen Lebensumständen an- 
geborene Neigung und Fähigkeit zu der späteren Wirksamkeit entfaltet haben.

Georg Treffer ist am 11. April 1847 im Dorfe Luttach, am südlichen Fusse des Schwarzenstein, im Taufererthale gelegen, zur Welt gekommen.

Seine Mutter war als Hebamme thätig und versuchte sich auch nach Art mancher ihrer Berufsgenossinnen in der Ausübung bäuerlicher Arzneikunde.

Damit war selbstverständlich die Beschäftigung mit heilkräftigen oder als solchen angesehenen Kräutern verbunden und das Interesse für die Pflanzenwelt schon von frühester Jugend an im Knaben geweckt, der die Mutter zu derartigen Zwecken in's Gebirge begleitete und gar bald selbst für sie das Gewünschte herbeischaffte.

Seine Schulbildung beschränkte sich auf den Unterricht der beimatlichen Volksschule. Das, zumal in jener Zeit, mehr als dürftige Mass derselben trachtete er jedoch bald durch Lectüre zu ergänzen. Bei seiner stillen, zurückgezogenen Lebensweise fand er auch weder Geschmack am Wirthshausbesuche noch an dem Umgange mit anderen Burschen seines Alters, was ihm den Spitznamen "der hearische Jorgl" einbrachte.

Im Winter 1872-1873 kam er bei Gelegenheit der Erstattung von Krankenberichten für eine Verwandte in Beziehungen zu dem bekannten Erforscher der Zillerthaler Eiswelt, Dr. Josef Daimer. damals praktischen Arzt in Taufers, jetzt k. k. Ministerialrath und Mitglied des obersten Sanitätsrathes in Wien.

Das ganz ungewornnliche Interesse des jungen Mannes für die Natur, sein hochst bescheidenes Wesen bei auf dem Lande nur selten anzutreffenden gefälligen Manieren veranlasste Dr. Daimer, demselben grơssere Aufmerksamkeit zu widmen und ihn zum Bergführer heranzubilden. Bei den zahlreichen zu diesem Behufe unternommenen Touren stellte sich bereits deutlich seine Neigung zum Pflanzensammeln heraus und hier war es, wo er unter der Anleitung seines auch in der Botanik bewanderten Grnners sich die ersten floristischen Kenntnisse aneignete.

Schon im Jahre 1873 sehen wir Treffer als Mitglied der damals von Daimer gegründeten Section Taufers des Deutschen und Oesterreichischen Alpenvereins und im Jahre 1874 als autorisierten Bergführer. Letzteres gab ihm Gelegenheit, Bekanntschaften anzuknüpfen, welche ihm den Gedanken an eine Verwertung seiner Liebhaberei durch Export nahelegten, und so war es zunächst die Einsammlung grosser Mengen yon Achillea moschata, wodurch er bei einer Münchener Liqueurfabrik Verdienst erlangte.

Bald nachher hatte er das Glück, weitere Anregung und Belehrung speciell im naturwissenschaftlichen Sinne von einer anderen Seite zu erhalten, dem jetzigen Stiftscanonicus in Innichen, Leonbard Wiedemayr. Derselbe war so freundlich, hierüber Nachstehendes mitzutheilen: 
"Als ich zu Anfang des September 1877 als Cooperator in Luttach eintrat, stellte sich Treffer mir vor, da er von meiner Vorliebe für Botanik Kenntnis hatte, und bat mich, ihm in der weiteren Ausbildung in diesem Fache behilflich zu sein, was meinerseits bereitwilligst zugesagt wurde. Ich staunte billig über seine Kenntnisse, die er damals schon besass; es ist indessen meinem Gedächtnisse entschwunden, woher er die ersten Anfänge sch⿰píte. Gewiss ist, dass Treffer wiederholt als Bergführer mit Herren aus verschiedenen Ländern in's Gebirge ging, eifrig Alpenpflanzen sammelte, sich unverdrossen um deren Namen kümmerte und diese sodann in den Büchern, die er sich mittlerweile angeschafft oder von mir entlehnt hatte hauptsächlich war es Hausmann's Flora von Tirol], nachschlug. Wo immer er einen Freund der Botanik wusste, setzte er sich mit ihm in's Einvernehmen, bald personlich, bald auf schriftlichem Wege. Während meines zweijährigen Aufenthaltes in Luttach verkehrten wir oft mitsammen, theilten uns gegenseitig die botanischen Erlebnisse und Kenntnisse mit, besichtigten die Erfolge unserer Excursionen u. s. w. Einmal nahm ich Einsicht in sein Herbar und fand dasselbe in Bezug auf Determination fast durchwegs in Ordnung. Lateinische Namen musste ich ihm wiederholt erklären, wobei sein Notizbuch arg mitgenommen wurde. Als ich 1878 mit dem Studium der Conchylien begann, lauschte er mir Manches ab, gewann Tiebe auch für dieses Fach. sammelte mit gewohntem Eifer, setzte sich mit S. Clessin in's Einvernehmen und hatte bald eine ansehnliche Schneckensammlung. Nach meiner Versetzung nach Taufers und Pfitsch blieben wir in Correspondenz, bis mich mein Beruf als Religionslehrer in Innsbruck dieselbe abbrechen hiess, da ich, die Ferienmonate abgerechnet, nicht mehr Zeit und Gelegenheit zum Studium der Naturgeschichte fand."

Im Jahre 1880, zur nämlichen Zeit, als Dr. Daimer durch Ernennung zum k. k. Sanitätsassistenten in Bludenz seinem alpinen Wirken im heimatlichen Thale entzogen wurde, kam Treffer mit Rupert $\mathrm{Huter}$ in Contact, und zwar geschah dies auf einer vereinbarten Excursion durch das Ampezzanerthal. Im darauffolgenden Sommer botanisierte er unter seiner Führung eine Woche im Kalserthale und 1885 begleitete er ihn auf einer Reise in die karnischvenetianischen Alpen.

Nachdem er so von den reichen Erfahrungen des Meisters, speciell in der Technik des Präparierens grosserer Vorräthe ausgiebigen Nutzen gezogen und durch seine Vermittlung weitere $A b$ satzquellen erlangt hatte, begann er auch, während er sich von dem nur wenige Jahre ausgeübten Bergführerberufe zurückzog, für grőssere wissenschaftliche Kreise zu arbeiten. Schon 1881 versendete er - noch mit geschriebenen Etiquetten - Pflanzen (Phanerogamen, Pteridophyten und nebenbei auch Moose) nach vorher ausgegebenen Listen, deren Druck ihm lange Zeit hindurch Huter besorgte, wofür er aber in den letzten Jahren selbst eine 
Presse anschaffte. Später erhielt er auch (durch Huter) Exsiccaten von Porta und Rigo im Austausch, ebenso Vieles von anderer Seite, und nach 1898 übernahm er Rig 0's Sammlungen in Commission.

So sehen wir ihn, wenn auch der Unternehmungsgeist zu grösseren Reisen offenbar nicht seine Sache gewegen ist, nahezu drei Jahrzehnte hindurch beschäftigt, den Pflanzenschätzen des Taufererthales in seinen mannigfachen $\nabla$ erzweigungen, worunter an erster Stelle das Weissenbach- und Mühlwaldthal, dann die Thäler Trippach und Rein zu nennen sind, nachzuspüren und sie in reicher Fülle, prächtig getrocknet oder als lebendes Gartenmaterial aller Welt, namentlich aber nach dem Deutschen Reiche, zu vermitteln.

Diese Thätigkeit erwies sich auch bei seinen geringen $\mathrm{Be}-$ dürfnissen materiell als lohnend, so dass er sich verheiraten (1889) und mit Hilfe eines Verwandten ein kleines, nettes Haus in Luttach bauen konnte. Dabei war er, wie Herr Ministerialrath Dr. Daim er weiter mitzutheilen die Güte hatte, auch in späteren Jahren stets derselbe geblieben, schüchtern, fast scheu, ganz seinem Arbeitsfelde zugewandt, das ihm als weiteren Vulgonamen die Bezeichnung "Wurzenkramer" eintrug, und von trener Fürsorge um seine Familie erfüllt.

Im Frühling 1901 begannen sich die ersten Zeichen der Krankheit fühlbar zu machen, welche den wackeren Mann im Verlaufe von einundeinhalb Jahren noch im kräftigsten Alter dahinraffen sollte.

Gleichwohl ging er noch in jenem Sommer seiner gewohnten Thätigkeit nach: "auf Sammlung", wie man sich in Luttach ausdrückte, allein es sollte das letzte Mal sein. Ueber die Art und den Verlauf der Todesursache verdanke ich dem behandelnden Arzte, Herrn Dr. Jacob Erlacher in Taufers, folgende Nachricht: "Das Leiden, welches in der letzten Zeit ein sehr qualvolles war, bestand hochstwahrscheinlich in einer Neubildung im oberen Mittelfellraume der Brust. Durch eine Section konnte allerdings die Diagnose nicht bestätigt werden. Im Vordergrunde der Erscheinungen standen Anfangs Herzbeschwerden, zu denen sich später neben Athembeschwerden und constanter Heiserkeit Bluthusten und starke Schmerzen in der Brust gesellten."

Als ich am 24. Mai 1903 das am Rande eines kleinen Lärchengehőlzes ober der Kirche von Luttach gelegene Haus des Verstorbenen mit seinen gemüthlichen, zirbenholzgetäfelten Räumen aufsuchte, in Sauberkeit und Accuratesse ganz den mitgebrachten Vorstellungen entsprechend, da fand ich die schwergeprüfte, mit drei aufgeweckten Knaben im Alter von 12, 7 und $1^{1 / 2}$ Jahren ihrer Stütze beraubte Witwe, sowie den herbeigerufenen Vormund, Herrn Lehrer Alfons S e eber, mit grosster Zuvorkommenheit bereit, nach Moglichkeit Auskünfte über den Verstorbenen zu ertheilen und in dessen wissenschaftlichen Nachlass Einsicht zu gewähren. 
Letzterer besteht in Folgendem:

1. Dem Herbar, auf Halbbogen kleineren Formats autgespannt, in 27 Fascikel à 70-80 Exemplare vertheilt, somit bei 2000 Exemplare zählend.

2. Dem Doublettenvorrath, mit Exemplaren von Huter, Porta, Cimarolli u. A., 23 Fascikel mit durchschnittlich 37 Arten in je ungefähr 10 Exemplaren, zusammen etwa 8500 Exemplare zählend, noch tadellos erhalten (nach Abrechnung von 600 Exemplaren, welche an G. Rig 0 zurückzustellen sind).

3. Einer Conchyliensammlung mit 793 tirolischen und aussertirolischen Arten, durchgehends bestimmt und mit Fundortsangaben versehen.

4. Einer kleinen, sorgfältig gehaltenen Bibliothek.

Ueberdies fand sich noch vor eine Anzahl Alpenpflanzen in Gartencultur.

Frau Maria Treffer will die beiden systematischen Sammlungen, sowie die Bücher für den Fall behalten, dass sich etwa bei einem ihrer Sőhne die Neigung bekunden sollte, den Fusstapfen des Vaters zu folgen; dagegen wünscht sie, für die Doubletten, wenn auch um billigen Preis, einen Abnehmer zu finden, und zwar so bald als möglich, da deren Aufbewahrung nicht für längere Dauer eingerichtet ist.

Ludwig Graf v. Sarnthein, Innsbruck.

\section{Marsupella olivacea Spruce.}

In Nr. 5 des laufenden Jahrganges dieser Zeitschrift erörtert Schiffner den Bau der oben geuannten Pflanze und kommt nach Prüfung des Originalmaterials zu der Ueberzengung, dass diese Art zu Gymnomitrium adustum Nees zu stellen, der Name also einzuziehen sei. Er erwähnt dabei am Eingange seiner Abhandlung die weitläufige Beschreibung des Autors und bemerkt, dass dieselbe allen Hepaticologen ein Räthsel geblieben sei.

In der That ist aus dieser Diagnose Spruces nicht zu ersehen, ob die Pflanze ein Gymnomitrium oder eine Marsupella ist. Mir hat es immer geschienen, dass seine Diagnose zwei verschiedenen Arten entnommen sei und dass Spruce, unter dem Einflusse seiner bekannten Anschauung, dass beide Gattungen zu einer vereinigt werden müssen, zu einer klaren Unterscheidung der in seinem Material enthaltenen Pflanzen nicht gelangt ist.

Das Material, welches meiner Publication (Spec. Hepat. II, p. 18) zur Unterlage diente, empfing ich von Stabler selbst, der dasselbe am Bow Fell gesammelt hat, also an demselben Standorte, von welchem Schiffner's Material staminte.

Schon dieser, Umstand lässt meine obige Vermuthung berechtigt erscheinen. 